\title{
Clinical relevance of lysyl oxidase-like 2 and functional mechanisms in glioma
}

This article was published in the following Dove Press journal:

OncoTargets and Therapy

\author{
Xiao-Guang Du ${ }^{1,2}$ \\ Mei-Jia Zhu' \\ 'Department of Neurology, \\ Qianfoshan Hospital Affiliated \\ to Shandong University, Jinan, \\ Shandong, People's Republic of China; \\ ${ }^{2}$ Department of Neurology, Weifang \\ People's Hospital, Weifang, Shandong, \\ People's Republic of China
}

Introduction: Glioma is the most frequent malignancy of the adult central nervous system with high recurrence risk and poor prognosis. Understanding the biological molecular mechanisms involved in glioma progression is critical for studying oncogenic mechanisms and improving prognosis. Lysyl oxidase-like 2 (LOXL2) is a kind of lysyl oxidase catalyzing the formation of peptidyl-lysine residues and promoting intramolecular cross-linking, especially for proteins in extracellular matrix. Our study explored the expression pattern of LOXL2 in glioma for the first time and found that its high expression was associated with larger tumor size and advanced tumor grade $(P<0.05)$. Moreover, univariate and multivariate analyses revealed LOXL2 as a novel independent prognostic factor for the overall survival of glioma patients.

Methods: To evaluate the detailed functional roles of LOXL2, we tested its oncobiology characteristics in U87-MG cells with overexpression and knockdown experiments.

Results: Cellular results demonstrated that LOXL2 overexpression enhanced cell proliferation and invasion, while LOXL2-siRNA attenuated cell viability. Furthermore, our data identified the participation of E-cadherin, Snail1, Src, and FAK proteins downstream of LOXL2. Notably, by using immunoprecipitation and mass spectrometry strategies, we initially verified the interaction between LOXL2 and HDAC2, indicating the existence of a protein complex containing LOXL2/Snail1/HDAC2. Additionally, the expression of HDAC2 protein was highly correlated with that of LOXL2 in clinical glioma tissues $(P=0.02)$, further implying the synergic oncogenic roles of these 2 proteins.

Conclusion: LOXL2 is a promising prognostic biomarker and may be further evaluated as a potential drug target for patients with glioma.

Keywords: glioma, HDAC2, invasion, LOXL2, prognosis

\section{Introduction}

Glioma is the most frequent primary brain malignancy of adults and is characterized by quick progression and poor survival. ${ }^{1,2}$ According to the World Health Organization (WHO), glioma can be classified into pilocytic astrocytoma (grade I), diffuse astrocytoma (grade II), anaplastic astrocytoma (grade III), and glioblastoma (grade IV). ${ }^{3}$ Surgical resection is the most effective treatment for glioma; meanwhile, radiotherapy and chemotherapy development have led to significant progress. However, the clinical outcomes of glioma patients are far from satisfactory, with more than half of the patients dying within 2 years after diagnosis due to, inevitably, postoperative recurrence. ${ }^{4}$ One of the major prognostic factors is the tumor grade, and it is widely used in clinical prognosis. Accumulating studies have shown that even patients suffering from the same tumor type and at the same tumor grade may have completely different prognosis. ${ }^{5}$ Therefore, an understanding of the biological and molecular factors involved in the progression of glioma is critical for further improvement in prognosis predication and therapy selection. ${ }^{6}$ 
Recent evidence revealed that the extracellular matrix $(E C M)$ plays critical roles in modulating tumor growth and metastasis. ${ }^{7}$ The biological change in ECM can directly regulate cell adhesion and dissemination, and thus more attention is focused on studying the role of ECM-related proteins in tumor progression. For example, the lysyl oxidase (LOX)like 2 (LOXL2), a member of the LOX family, is a kind of secreted, copper-dependent amine oxidase. ${ }^{8}$ LOXL2 contains a copper-binding motif and a lysyl-tyrosyl-quinone group in its C-terminus, ${ }^{9}$ and thus it can oxidatively deaminate the peptidyl-lysine residues and generate reactive aldehyde groups to initiate covalent inter- and intramolecular cross-linking. ${ }^{10}$ The ECM protein collagen and elastin are well-known substrates of LOXL2. ${ }^{11}$ Additionally, it has been reported that E-cadherin was downregulated by LOXL2, resulting in enhanced epithelial-mesenchymal transition process. ${ }^{12}$ Although, the above studies implied the possible involvement of LOXL2 in tumor progression, its expression pattern and clinical significance in glioma have not been illuminated.

Here in this study, we explored the expression and prognostic role of LOXL2 in glioma patients for the first time. Besides, we characterized the role of LOXL2 in promoting glioma cell proliferation and invasion using U87-MG glioma cell line. Importantly, mass spectrometry (MS) and immunoprecipitation experiments verified the interaction between LOXL2 and Snail1/HDAC2 proteins, providing novel insights into the functional mechanisms of LOXL2 in tumor progression.

\section{Patients and methods}

\section{Patients and tissue samples}

We randomly enrolled 65 paraffin-embedded glioma tissues obtained from Qianfoshan Hospital Affiliated to Shandong University. Another 8 freshly isolated normal brain tissues and 13 glioma tissues were collected and stored at $-80^{\circ} \mathrm{C}$ until use. None of the above patients had received chemotherapy or radiotherapy prior to surgery. All diagnoses were based on histological examination by the Department of Pathology according to the WHO criteria. This study was approved by the Human Ethics Committee of Qianfoshan Hospital. Written informed consent authorizing the collection and use of tissue samples for research purposes was obtained from all patients. The patients were retrospectively followed up, and the retrieved clinicopathological characteristics are summarized in Table 1.

\section{Immunohistochemically (IHC) staining and $\mathrm{IHC}$ evaluation}

To determine the protein expression and cellular localization of LOXL2, a $4 \mu \mathrm{m}$ section of each tumor specimen was
Table I Correlations between LOXL2 level and clinical parameters of glioma patients

\begin{tabular}{|c|c|c|c|c|}
\hline \multirow[t]{2}{*}{ Variables } & \multirow{2}{*}{$\begin{array}{l}\text { Cases } \\
(n=65)\end{array}$} & \multicolumn{2}{|c|}{ LOXL2 expression } & \multirow[t]{2}{*}{$P$-value } \\
\hline & & $\begin{array}{l}\text { Low } \\
(n=42)\end{array}$ & $\begin{array}{l}\text { High } \\
(n=23)\end{array}$ & \\
\hline \multicolumn{4}{|l|}{ Gender } & 0.418 \\
\hline Male & 41 & 28 & $13(31.70 \%)$ & \\
\hline Female & 24 & 14 & $10(41.67 \%)$ & \\
\hline \multicolumn{4}{|l|}{ Age (years) } & 0.174 \\
\hline$\leq 50$ & 35 & 20 & $15(42.86 \%)$ & \\
\hline$>50$ & 30 & 22 & $8(26.67 \%)$ & \\
\hline \multicolumn{4}{|l|}{ Tumor size } & $0.006 *$ \\
\hline$\leq 5 \mathrm{~cm}$ & 32 & 26 & $6(18.75 \%)$ & \\
\hline$>5 \mathrm{~cm}$ & 33 & 16 & 17 (5।.52\%) & \\
\hline \multicolumn{4}{|l|}{ WHO grade } & $0.039 *$ \\
\hline II & 31 & 24 & 7 (22.58\%) & \\
\hline III-IV & 34 & 18 & $16(47.06 \%)$ & \\
\hline \multicolumn{4}{|c|}{ Karnofsky score } & 0.652 \\
\hline$\leq 90$ & 40 & 25 & $15(37.50 \%)$ & \\
\hline$>90$ & 25 & 17 & $8(32.00 \%)$ & \\
\hline
\end{tabular}

Note: *Statistically significant by $\chi^{2}$-test.

Abbreviations: LOXL2, lysyl oxidase-like 2; WHO, World Health Organization.

subjected to IHC analysis. The slides were deparaffinized, rehydrated, and treated with $3 \% \mathrm{H}_{2} \mathrm{O}_{2}$ in methanol for $15 \mathrm{~min}$ to inhibit endogenous peroxidase. After blocking with $10 \%$ nonimmunogenic goat serum at room temperature for $30 \mathrm{~min}$, slides were incubated with rabbit anti-LOXL2 polyclonal antibody (ab96233, Abcam, Cambridge, UK) overnight at $4^{\circ} \mathrm{C}$. PBS incubation was parallelly conducted as the negative control. Immunoreactivity was assessed with a 2-step assay kit (PV-6001, ZSGB-Bio, Beijing, People's Republic of China) and a DAB IHC detection kit (ZAI9017, ZSGB-Bio) according to the manufacturer's instructions. After counterstaining, dehydration, and mounting, 2 pathologists blinded to the clinical data were invited to evaluate the IHC results based on both staining proportion and intensity. The positive proportion was scored as 0 ( $0 \%-10 \%), 1(11 \%-25 \%), 2$ (26\%-50\%), 3 (51\%-75\%), and $4(75 \%-100 \%)$; the staining intensity was scored as 0 (negative), 1 (light yellow, weak), 2 (yellow, moderate), and 3 (dark brown, strong). The final IHC score was calculated by multiplying the 2 scores above. IHC score $<8$ was considered as low expression, while IHC score $\geq 8$ was grouped into high expression.

\section{RNA extraction and real-time quantitative PCR (RT-qPCR)}

Total RNA was extracted from tissues using Trizol reagent (Takara, Tokyo, Japan) according to the manufacturer's instruction. One microgram RNA was reverse-, and the 
cDNA product was used for RT-qPCR performed using the SYBR premix Ex Taq (Takara) on a Real-Time PCR Detection System with the following cycling program: $95^{\circ} \mathrm{C}$ for $10 \mathrm{~min}, 95^{\circ} \mathrm{C}$ for $15 \mathrm{sec}$ for 40 cycles, and $60^{\circ} \mathrm{C}$ for $1 \mathrm{~min}$. GAPDH was parallelly tested as an internal reference. The primers were as followed:

GAPDH, forward, 5'-ATGGGGAAGGTGAAGG TCG-3',

reverse, 5'-GGGGTCATTGATGGCAACAATA-3'; LOXL2, forward, 5'-ACATGTACCGCCATGACATC GACT-3', reverse, 5'-TGAAGGAACCACCTATGTGGCAGT-3'.

\section{Western blot}

The tissues or cells were lysed in RIPA buffer containing $50 \mathrm{mM}$ Tris- $\mathrm{HCl}$ (pH 7.5), $150 \mathrm{mM} \mathrm{NaCl}, 5$ mM EDTA, 0.5\% NP-40 (Sigma, Kawasaki, Japan), 5 mM dithiothreitol, $10 \mathrm{mM} \mathrm{NaF}$, protease, and phosphatase inhibitor cocktail (Thermo Fisher Scientific, Pittsburgh, PA, USA). The protein concentration was determined using a BCA assay kit (Biorad, Berkeley, CA, USA). Approximately $20 \mu \mathrm{g}$ protein was subjected to sodium dodecyl sulfate-polycrylamide gel electrophoresis and transferred to a PVDF membrane (Thermo Fisher Scientific). After blocking with 5\% nonfat milk at room temperature for $1 \mathrm{~h}$, the membrane was incubated with corresponding primary antibodies (LOXL2, Snail1, E-cadherin, phosphor-Src, Src, phosphor-FAK, FAK, HDAC2, and $\beta$-tublin) at $4^{\circ} \mathrm{C}$ overnight. Immunoblotting was conducted with another incubation with secondary antibodies conjugated to horseradish peroxidase. Immunoreactivity was visualized with X-ray film (Fujifilm, Tokyo, Japan) using ECL-Plus detection reagents (Santa Cruz Biotechnology, Santa Cruz, CA, USA).

\section{Cell culture and transfection}

Human normal cortical cell line ( $\mathrm{HCN}-2)$ and 3 different glioma cell lines (U87-MG, U251, and U118-MG) were obtained from the American Type Culture Collection (Shanghai, People's Republic of China). Cells were cultured in Dulbecco's Modified Eagle's Medium (Thermo Fisher Scientific) supplemented with 10\% fetal bovine serum (Gibco, Life Technologies, Carlsbad, CA, USA) and maintained at $37^{\circ} \mathrm{C}$ in a humidified chamber containing $5 \% \mathrm{CO}_{2}$.

HA-tagged LOXL2, GFP-tagged LOXL2, and YFPtagged Snail1 plasmids in pCDNA3.1 vector were constructed by Sangon Biotech (Shanghai, People's Republic of China) and confirmed by DNA sequence. For knockdown experiments, siRNA was synthesized by GenePharma (Shanghai, People's Republic of China) with the following sequence: LOXL2: 5'-CAGUCUAUUAUAGUCACAU-3'; negative control: 5'-UUCUCCGAACGUGUCACGU-3'. Both overexpression and knockdown were performed by transient transfection with FuGENE ${ }^{\circledR} 6$ Transfection Reagent (Promega, Madison, WI, USA) following the manufacturer's instructions. The transfection efficiency was tested by Western blot. Colocalization between transfected proteins were visualized via a fluorescence inversion microscope system.

\section{CCK-8 proliferation assay}

At $24 \mathrm{~h}$ after transfection, cells were seeded into 96-well plates $\left(5 \times 10^{3}\right.$ cells per well) and cultured at $37^{\circ} \mathrm{C}$ with $5 \% \mathrm{CO}_{2}$. At $1,2,3$, and 4 days, $10 \mu \mathrm{L}$ of CCK-8 solution (Beyotime, Beijing, People's Republic of China) was added into each well. Following incubation for $1 \mathrm{~h}$ at $37^{\circ} \mathrm{C}$, the absorbance of each well was measured at $450 \mathrm{~nm}$ wavelength. The experiment was performed in triplicate and repeated thrice.

\section{Migration and invasion assay}

Transwell chambers (Corning Inc., Corning, NY, USA) with or without Matrigel (BD Bioscience, San Jose, CA, USA) coating were used to perform migration and invasion assays, respectively. ${ }^{13}$ Briefly, transfected cells were cultured in serum-free medium in the upper chambers $\left(5 \times 10^{5}\right.$ cells per chamber), while the lower chamber contained $10 \%$ fetal bovine serum-supplemented medium. After $48 \mathrm{~h}$ of culture, Transwell membranes were fixed with methanol and stained with crystal violet. Noninvaded cells on the upper side of the membrane were gently wiped off, and the stained cells on the lower side were quantified under a microscope. The average number of invaded cells was calculated by counting 5 visual fields per chamber. Three independent experiments were conducted in triplicate.

\section{Immunoprecipitation and MS}

To identify interaction partners of LOXL2, we performed HA-affinity immunoprecipitation for U87-MG cells transfected with HA-LOXL2 plasmid. Briefly, transfected cells were harvested and lysed in lysis buffer containing $50 \mathrm{mM}$ HEPES (pH 7.5), 150 mM NaCl, 1 mM EDTA, 50 mM NaF, $1.5 \mathrm{mM} \mathrm{Na}_{3} \mathrm{VO}_{4}, 10 \mathrm{mM} \beta$-glycerophosphate, and protease inhibitor cocktail. After centrifugation at $13,000 \times g$ at $4{ }^{\circ} \mathrm{C}$ for $15 \mathrm{~min}$, supernatants were mixed with HA-affinity beads (Santa Cruz Biotechnology) and incubated overnight at $4^{\circ} \mathrm{C}$. HA-affinity beads were then washed with lysis buffer thrice. Finally, the binding proteins were eluted with synthesized HA peptide ( $1 \mathrm{mg} / \mathrm{mL})$, which were subjected to $\mathrm{MS}$ analysis and Western blot. 
For MS analysis, the immunoprecipitated samples were digested with trypsin and tested using shotgun strategy using a tandem MS Q Exactive ${ }^{\mathrm{TM}}$ Plus (Thermo Fisher Scientific) coupled online to an ultra performance liquid chromatography system. For MS scans, the m/z scan range was set from 250 to 1,500 . The obtained MS/MS data were processed using the Mascot search engine (Matrixscience, London, UK) and searched against SwissProt_human database as well as the reverse decoy database.

\section{Statistics}

All statistical analyses were performed using SPSS 20.0 (IBM Corporation, Armonk, NY, USA). The correlations between expression levels of LOXL2 and patients' characteristics were tested by $\chi^{2}$-test. Survival analysis and univariate analysis were assessed by Kaplan-Meier method and compared by log-rank test. Multivariate Cox regression analysis was used to identify independent prognostic factors using a forward stepwise approach. $P<0.05$ was considered statistically significant.

\section{Results}

\section{Patient characteristics}

The entire cohort contained 41 male and 24 female patients, with a median age of 49 years. Thirty-one patients $(47.69 \%)$ were suffering from WHO grade II, 22 (33.85\%) had grade III, and $12(18.46 \%)$ had grade IV tumor. The information on tumor size and Karnofsky performance score was also retrieved (Table 1). The median overall survival time was 41 months (range, 4-74 months), and there were 12 survivors by the end of follow-up.

\section{High expression of LOXL2 is associated with advanced tumor progression}

LOXL2 showed low and negative staining in normal brain tissues (Figure 1A). In glioma tumor cells, LOXL2 protein mainly located in the cytoplasm and ECM, but also showed detectable staining in the nucleus (Figure 1B). By RT-qPCR, we found that LOXL2 mRNA was expressed higher in glioma tissues than that in normal brain tissues (Figure 1C). Simultaneously, we evaluated its protein level in fresh resected tumor tissues, revealing an elevated protein expression than in normal brain tissues (Figure 1D).

Thus, we were interested in exploring whether higher LOXL2 was associated with glioma progression. By subgrouping patients into low LOXL2 group and high LOXL2 group, $\chi^{2}$-test showed that LOXL2 was positively correlated with tumor size $(P=0.006$, Table 1$)$. Additionally, higher immunoreactivity was seen in grade III and grade IV tumors
$(P=0.039)$, compared with grade II. There was no significant correlation between LOXL2 protein expression and patient gender, age, or Karnofsky score (Table 1).

\section{LOXL2 is an independent prognostic factor in glioma patients}

Given that a significant statistical association between LOXL2 level and disease progression was observed, we plotted Kaplan-Meier survival curves according to different clinicopathological characteristics (Figure 2). By univariate regression analysis, we identified that higher LOXL2 protein expression was an unfavorable parameter affecting patients' survival (mean survival time 29.11 \pm 4.71 vs $47.75 \pm 3.18$ months, $P=0.003$, Table 2 ), confirming the association between LOXL2 and tumor progression. Other conventional prognostic factors included tumor size and WHO grade ( $P=0.048$ and $P=0.001$, respectively).

To further explore the clinical significance of LOXL2, we performed multivariate Cox regression analysis with tumor size, WHO grade, and LOXL2 expression level (Table 3). Among them, advanced WHO grade led to a significantly worse prognosis with HR of $2.813(95 \%$ $\mathrm{CI}=1.561-5.069, P=0.001)$. Besides, LOXL2 also acted as an independent prognostic factor in glioma patients $(\mathrm{HR}=2.119,95 \% \mathrm{CI}=1.189-3.776, P=0.011)$. Tumor size showed no statistical significance in terms of multivariate analysis.

\section{LOXL2 promotes proliferation, migration, and invasion of U87-MG cells}

Since LOXL2 overexpression was observed in glioma tissues, our next question was whether LOXL2 had a direct functional role in facilitating glioma growth or metastasis. By testing the endogenous protein expression levels of LOXL2 in a normal cortical cell line (HCN-2) and in 3 different glioma cell lines (U87-MG, U251, and U118-MG), we found that LOXL2 was significantly higher expressed in U87-MG and U118-MG cells compared to normal HCN-2 cells (Figure 3A). Due to the moderate expression level of LOXL2 in U87-MG cells, we chose this cell line to perform further silencing and overexpressing studies using the FuGENE 6 reagent. CCK-8 assay indicated that LOXL2 knockdown significantly inhibited cell proliferation, while LOXL2 overexpression enhanced cell viability (Figure 3B). In addition, Transwell and Matrigel-Transwell analyses were performed to evaluate whether LOXL2 affected cell migration or invasion. Our results showed that overexpression of LOXL2 led to a significant increase of migrated as well as invaded cells, and silencing of LOXL2 attenuated these 
A

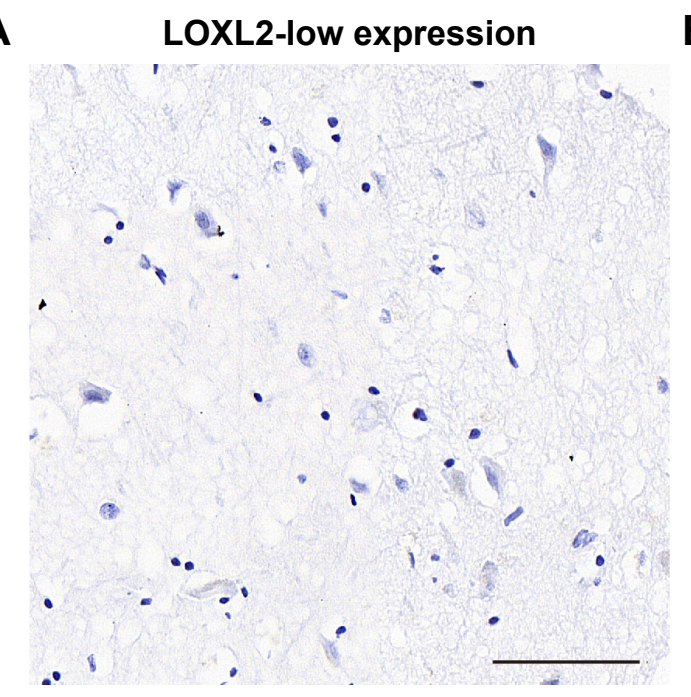

B LOXL2-high expression

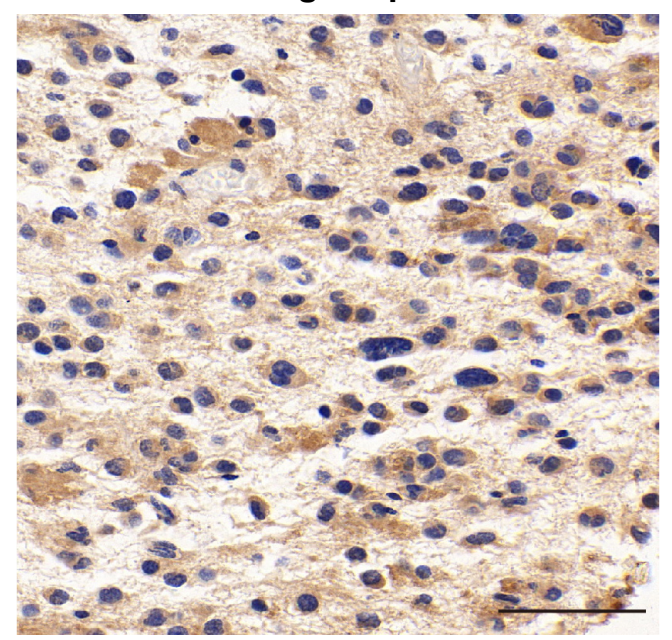

C

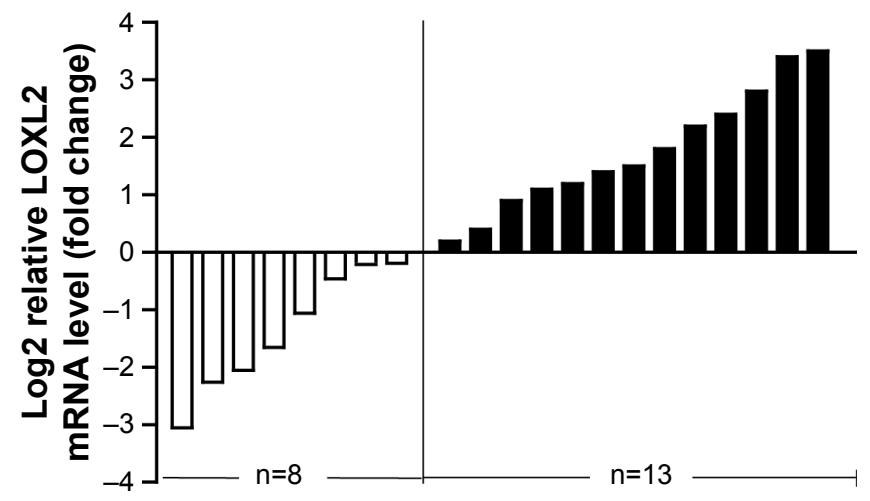

Normal tissue

Glioma tissue

D

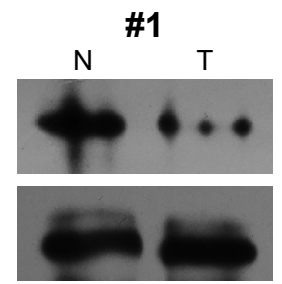

\#5

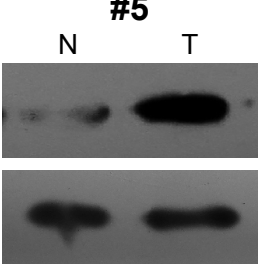

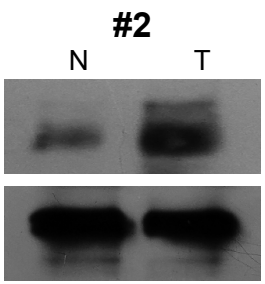

\#6

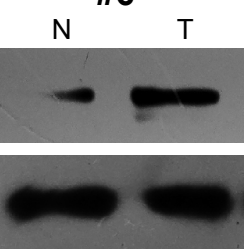

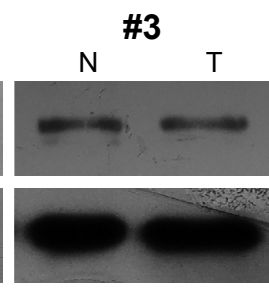

\#7

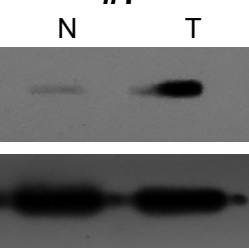

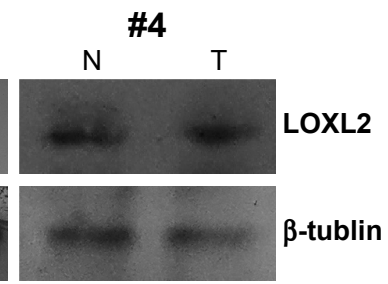

\#8



Figure I LOXL2 shows a higher expression level in glioma tissues.

Notes: (A) Representative negative staining of LOXL2 in normal brain tissue. (B) Representative high expression of LOXL2 in glioma tissue (male, 22 years old, WHO grade III). (C) RT-qPCR results of LOXL2-mRNA expression in normal brain tissues $(n=8)$ and glioma tissues ( $n=13$ ), showing a higher LOXL2-mRNA level in glioma $(P<0.05)$. (D) The protein expression of LOXL2 was tested in 8 paired freshly resected normal brain tissues $(N)$ and glioma tissues $(T)$ by Western blot, and glioma LOXL2 showed a higher protein level than normal brain tissues in 75.0\% (6/8) paired samples. Magnification 400x. Scale bar, $50 \mu \mathrm{m}$.

Abbreviations: LOXL2, lysyl oxidase-like 2; RT-qPCR, real-time quantitative polymerase chain reaction; WHO, World Health Organization.

processes (Figure 3C and D). These findings indicated that LOXL2 might play a tumor-promoting role in glioma.

Furthermore, Western blot analysis revealed that LOXL2 can inhibit E-cadherin level while upregulating Snail1 expression (Figure 3E), which was consistent with the results of another study. ${ }^{12}$ Interestingly, we also found that intracellular LOXL2 can positively regulate the phosphorylation levels of Src and FAK proteins without affecting the total protein levels. Similar signaling pathways were reported for secreted LOXL2 in gastric cancer. ${ }^{14}$ 




C Overall survival according
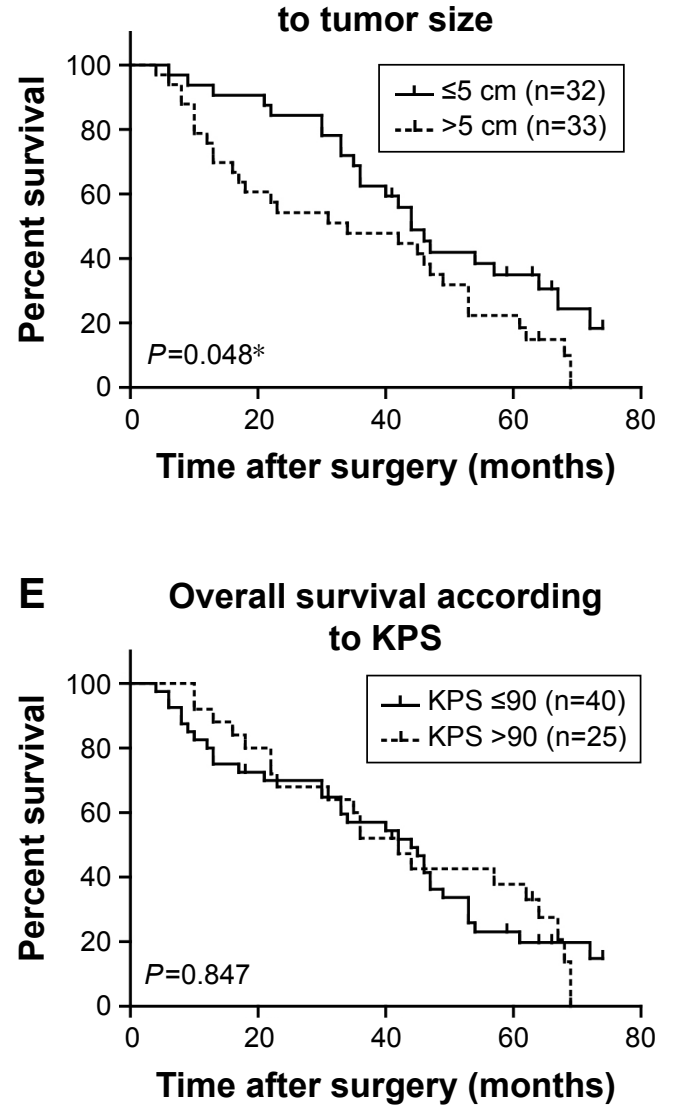

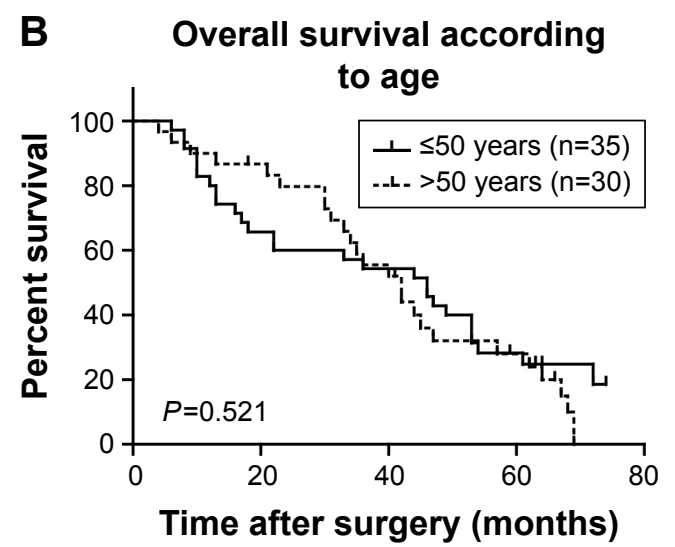

D Overall survival according
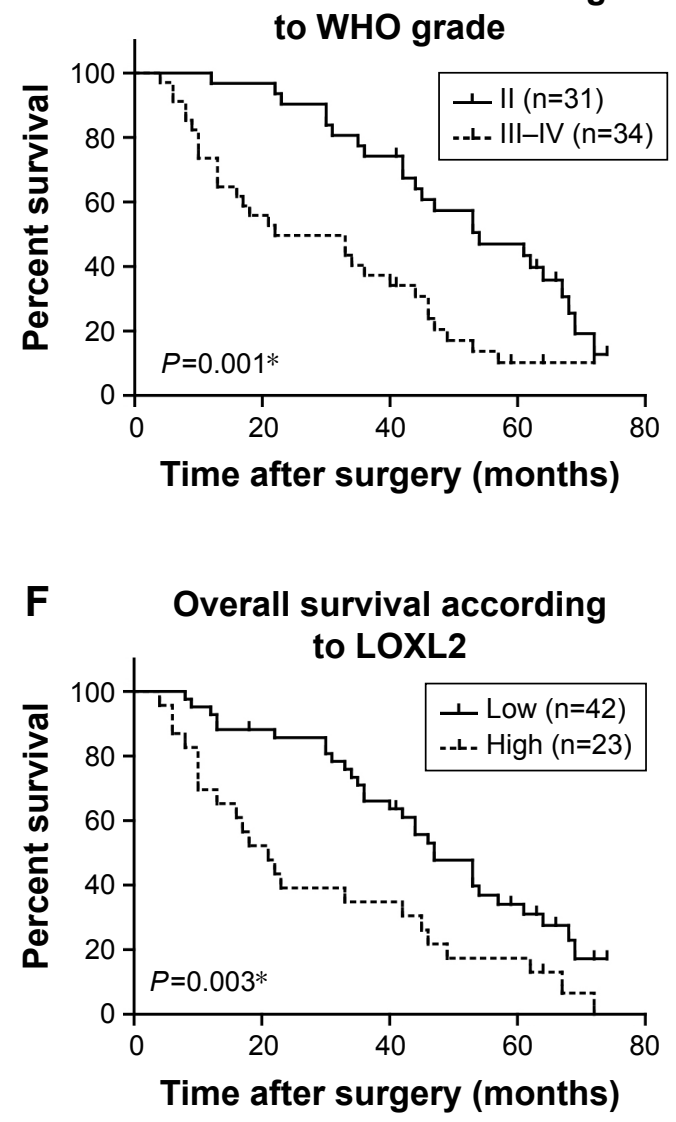

Figure 2 Kaplan-Meier survival curve according to different clinicopathological characteristics.

Notes: The overall survival analysis was performed based on patients' gender (A), age (B), tumor size (C), WHO grade (D), KPS score (E), and LOXL2 protein levels (F), respectively. The overall survival was evaluated by Kaplan-Meier univariate analysis and log-rank test. $* P<0.05$.

\section{LOXL2 downregulates E-cadherin by interacting and stabilizing Snail I/HDAC2 proteins}

To date, the mechanism by which LOXL2 regulates Snail1, E-cadherin, and other downstream proteins is unclear. We conducted MS experiments to detect the interactome of LOXL2 protein in U87-MG cells. By searching against
SwissProt_human database, we identified Snail1 protein and HDAC2 (Figure 4A) as novel binding partners of LOXL2. It has been reported that Snail1 can recruit HDAC1/HDAC2 complex and subsequently repress E-cadherin signaling in pancreatic cancer cells and HEK 293T cells. ${ }^{15-17}$ Therefore, we suspected that LOXL2 may also participate in Snail/ HDAC complex formation or stabilization. Consistent with 
Table 2 Univariate analysis for overall survival of glioma patients

\begin{tabular}{|c|c|c|c|c|}
\hline \multirow[t]{2}{*}{ Variables } & \multirow[t]{2}{*}{$\begin{array}{l}\text { Patients } \\
(n=65)\end{array}$} & \multicolumn{2}{|c|}{$\begin{array}{l}\text { Overall survival time } \\
\text { (months) }\end{array}$} & \multirow[t]{2}{*}{$P$-value } \\
\hline & & Mean \pm SD & Median & \\
\hline \multicolumn{4}{|l|}{ Gender } & 0.961 \\
\hline Female & 41 & $42.51 \pm 3.32$ & 45.00 & \\
\hline Male & 24 & $38.60 \pm 5.14$ & 34.00 & \\
\hline \multicolumn{4}{|l|}{ Age (years) } & 0.521 \\
\hline$\leq 50$ & 35 & $40.47 \pm 4.21$ & 46.00 & \\
\hline$>50$ & 30 & $41.69 \pm 3.76$ & 42.00 & \\
\hline \multicolumn{4}{|l|}{ Tumor size } & $0.048^{*}$ \\
\hline$\leq 5 \mathrm{~cm}$ & 32 & $42.27 \pm 3.74$ & 44.00 & \\
\hline$>5 \mathrm{~cm}$ & 33 & $35.08 \pm 4.04$ & 34.00 & \\
\hline \multicolumn{4}{|l|}{ WHO grade } & $0.001 *$ \\
\hline II & 31 & $52.36 \pm 3.28$ & 54.0 & \\
\hline III-IV & 34 & $30.59 \pm 3.69$ & 22.0 & \\
\hline \multicolumn{4}{|c|}{ Karnofsky score } & 0.847 \\
\hline$\leq 90$ & 40 & $40.03 \pm 3.70$ & 44.0 & \\
\hline$>90$ & 25 & $42.92 \pm 4.48$ & 42.0 & \\
\hline \multicolumn{4}{|c|}{ LOXL2 expression } & $0.003 *$ \\
\hline Low & 42 & $47.75 \pm 3.18$ & 52.0 & \\
\hline High & 23 & $29 .|I \pm 4.7|$ & 31.0 & \\
\hline
\end{tabular}

Note: *Statistically significant by log-rank test.

Abbreviations: LOXL2, lysyl oxidase-like 2; WHO, World Health Organization.

the MS data, immunoprecipitation experiments proved that LOXL2 can bind with Snaill and HDAC2 (Figure 4B).

Moreover, we tested the expression profile of HDAC2 protein in glioma tissues. The level of HDAC2 showed a significant positive correlation with LOXL2 level (Figure 4C, $P=0.02$ ). Intracellular interaction between HDAC2 and Snail1 was further verified by colocalization assay using fluorescence strategy (Figure 4D), thus indicating the existence of this protein complex in glioma cells (Figure 4E).

\section{Discussion}

LOXL2 was first reported as a collagen cross-linking enzyme, which can catalyze lysyl oxidation. ${ }^{18}$ Accumulating evidence showed the diverse cellular localization and functions of LOXL2. For example, it can cross-link the proteins in ECM by catalyzing oxidative deamination of peptidyl-lysine

Table 3 Multivariate analysis for the independent prognostic factors of glioma patients

\begin{tabular}{llll}
\hline Variables & HR & $\mathbf{9 5 \% ~ C l}$ & $P$-value \\
\hline Tumor size $(>5 \mathrm{~cm}$ vs $\leq 5 \mathrm{~cm})$ & $\mathrm{I} .699$ & $0.949-3.044$ & 0.075 \\
WHO grade (III-IV vs II) & 2.813 & $1.56 \mathrm{I}-5.069$ & $0.00 \mathrm{I}^{*}$ \\
LOXL2 expression (high vs low) & 2.119 & $1.189-3.776$ & $0.01 \mathrm{I}^{*}$ \\
\hline
\end{tabular}

Note: *Statistically significant.

Abbreviations: LOXL2, lysyl oxidase-like 2; WHO, World Health Organization. residues. ${ }^{19}$ Secreted LOXL2 also showed its effects by stimulating surrounding cells, such as by activating fibroblasts and promoting gastric cancer cell metastasis. ${ }^{20}$ Besides ECM, LOXL2 is also localized in the cytoplasm, where it regulates cell plasticity and polarity. ${ }^{8,21}$ In addition, LOXL2 plays roles in cell nucleus by modulating Snail-related signaling and transcriptional process. ${ }^{22}$ Importantly, LOXL2 shows tumor-promoting functions in gastric cancer, ${ }^{23}$ breast cancer, ${ }^{24}$ hepatocellular carcinoma, ${ }^{25}$ and lung squamous cell carcinoma. ${ }^{26}$ However, the high diversity of LOXL2 function may result in paradoxical effects in different tissues or cell types. ${ }^{27}$

Here in the current study, we initially found that the mRNA and protein levels of LOXL2 in clinical glioma tissues were higher than those in normal brain tissues. As demonstrated by statistical analysis, LOXL2 is an independent unfavorable biomarker for glioma patients. Since LOXL2 exhibited upregulated expression in patients with advanced tumor grade, we performed cellular studies to evaluate its oncogenic roles. Consistent with in other tumor types, LOXL2 overexpression significantly enhanced glioma tumor cell proliferation and invasion, indicating its potential as a novel drug target. Taking into consideration the fact that LOXL2 has complex functions, we next aimed to identify its global binding partners to better elucidate its signaling network.

HDAC1 and HDAC2 are well-known tumor suppression proteins. ${ }^{28}$ Previous studies showed the existence of a HDAC1/ HDAC2/Snail complex, which can inhibit E-cadherin signaling in pancreatic cancer. ${ }^{29}$ Besides HDAC1/HDAC2, Snail1 protein was also reported to be directly regulated by LOXL2, thus exerting transcriptional and epigenetic functions. ${ }^{30}$ Notably, we identified the existence of both Snal1 and HDAC2 in LOXL2 protein complexes by using immunoprecipitation and MS strategies. Further IHC data demonstrated a statistically significant correlation between HDAC2 and LOXL2 expression in glioma tissues. Therefore, it is high likely that LOXL2 can recruit HDAC2 to interact with Snaill, forming a functional heterotrimer, or even hereotetramer with the involvement of HDAC1, to inhibit E-cadherin signaling. However, whether and how this protein complex is assembled needs further structural information.

Last but not least, our data suggested another signaling pathway downstream of LOXL2, which is accomplished by phosphorylation of Src and FAK protein complex. Src and FAK proteins can both be activated upon phosphorylation, subsequently promoting cell proliferation via a wide range 
A

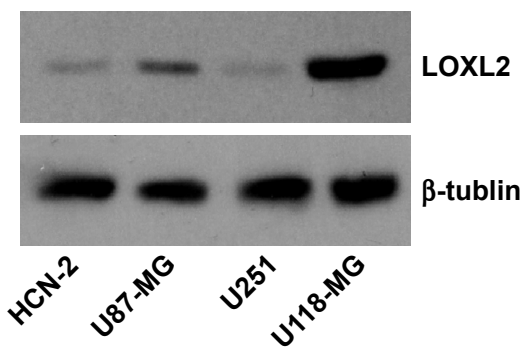

C

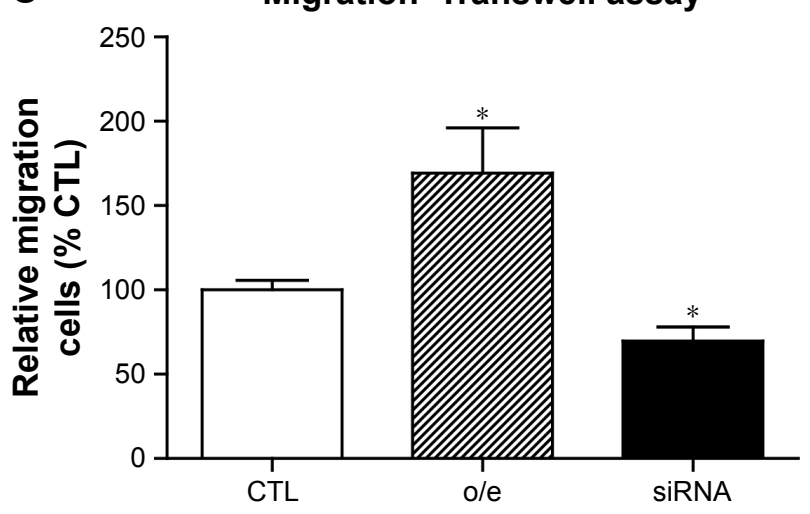

B

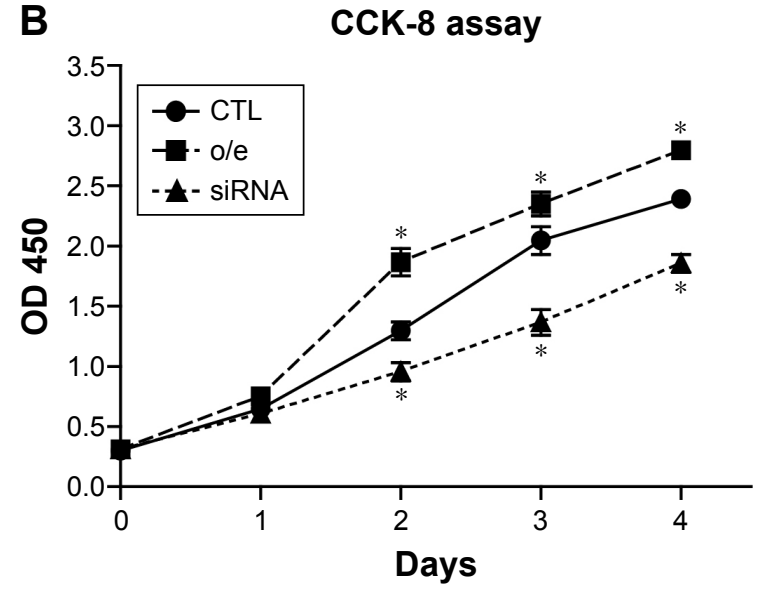

E

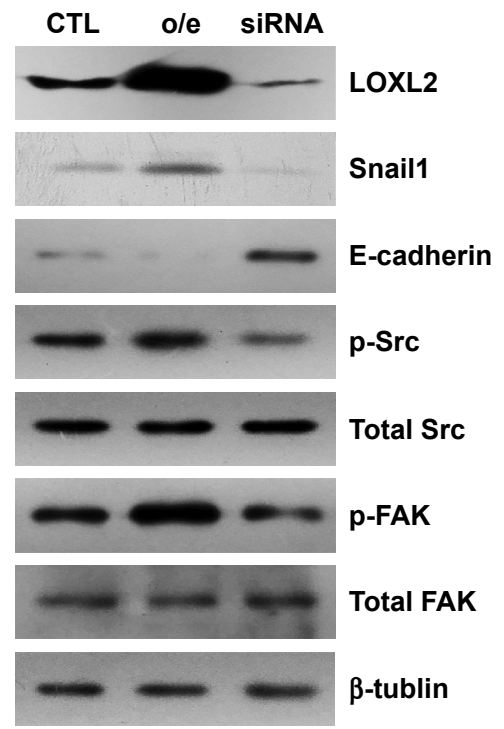

D Matrigel-Transwell assay

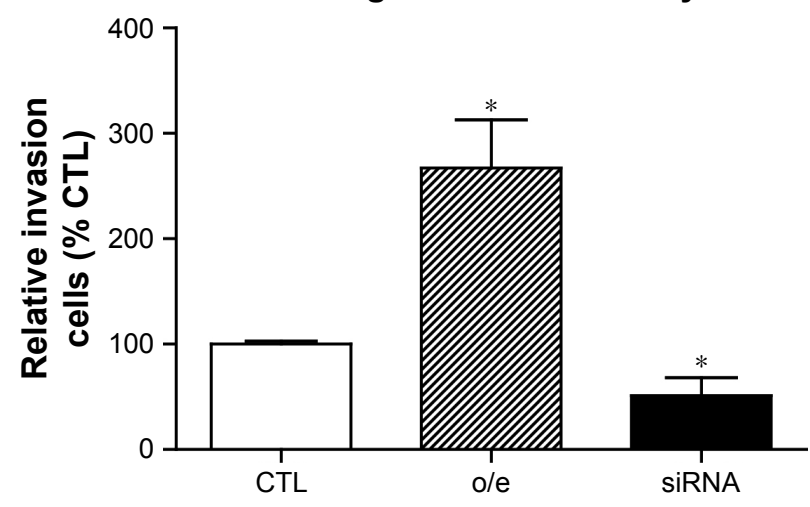

Figure 3 LOXL2 enhances proliferation and invasion capacities of glioma cells.

Notes: (A) Endogenous protein expression level of LOXL2 in normal cortical cell line (HCN-2) and 3 different glioma cell lines (U87-MG, U25I, and UI I8-MG) was tested by Western blot. (B) Proliferation of U87-MG cells was tested by CCK-8 assay, revealing a higher cellular viability in cells o/e LOXL2. In contrast, cells transfected with LOXL2-siRNA showed a decreased proliferation pattern. Migration-Transwell (C) and Matrigel-Transwell (D) experiments were performed to evaluate the migration and invasion processes of glioma cells. Similar to the proliferation results, LOXL2 expression level was positively associated with cell migration and invasion capacities. (E) Immunoblotting results revealed that LOXL2 can upregulate the protein expression of Snail I, while downregulating E-cadherin. Besides, the phosphorylation status of Src and FAK proteins was also enhanced by LOXL2 overexpression. Western blot results were semi-quantified using Image Software. All data were summarized from at least 3 independent experiments. $* P<0.05$ compared to $C T L$ group.

Abbreviations: CTL, control; LOXL2, lysyl oxidase-like 2; NS, not significant; o/e, overexpressing. 
A Snail1 peptide sequence: THTGEKPFSCPHCSR, charge: +3, m/z: $562.92224 \mathrm{Da}$

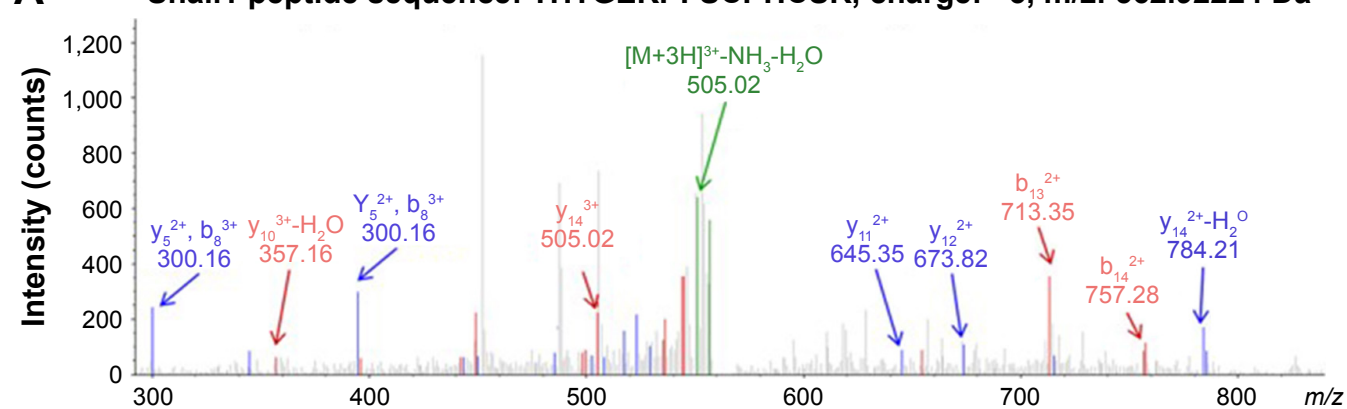

HDAC2 peptide sequence: SPVAAVAGEPMAYSQGGGKK, charge: +3, m/z: 635.65808 Da

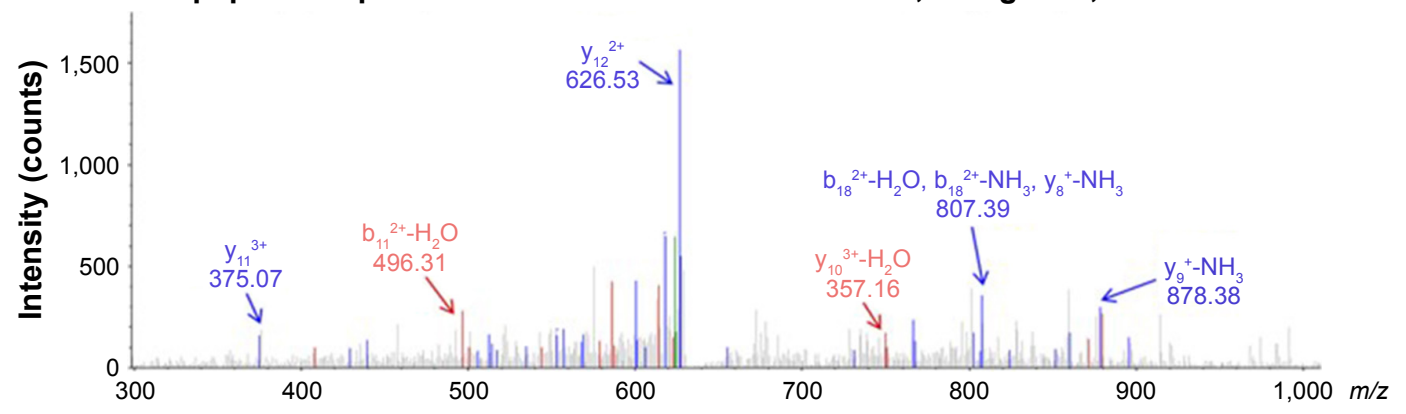

B

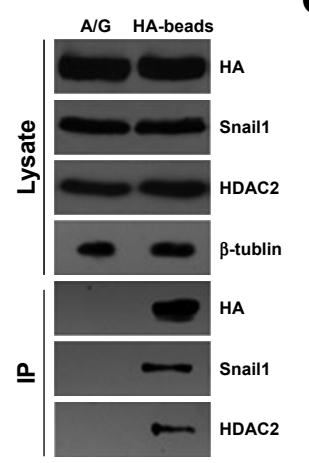

C


DAPI
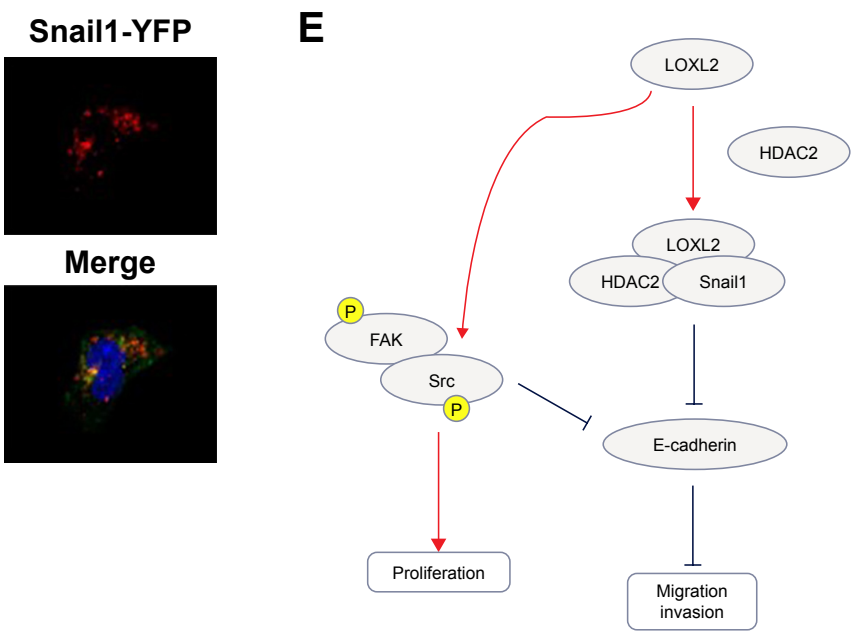

Figure 4 LOXL2 interacts with Snaill and HDAC2 proteins.

Notes: (A) Representative MS/MS spectrum showing identification of Snail protein and HDAC2 protein immunoprecipitated by LOXL2. The unique peptides were generated by tryptic digestion and mapped by a QE-plus UPLC-MS/MS instrument. Data was analyzed using Mascot method. (B) U87-MG cells transfected with HA-LOXL2 were subjected to HA-affinity agarose (HA-beads) immunoprecipitation, using protein A/G agarose as control (A/G). The immunoprecipitation data showed a significant interaction between LOXL2 and Snaill and HDAC2. (C) The protein expression of HDAC2 in glioma tissues was explored by IHC staining, demonstrating a positive correlation with the LOXL2 expression levels $\left(\chi^{2}\right.$-test, $\left.P=0.02\right)$. (D) Immunofluorescence assay demonstrated an obvious colocalization between LOXL2 and HDAC2 in the cytoplasm. (E) Schematic signaling pathways downstream of LOXL2 as revealed in our study. Magnification $400 \times$. Scale bar, $50 \mu \mathrm{m}$. $*$ Indicates $P<0.05$ by chi-square test. Abbreviations: IHC, immunohistochemistry; LOXL2, lysyl oxidase-like 2; MS, mass spectrometry; UPLC, ultra performance liquid chromatography. 
of molecular effectors. ${ }^{31}$ Therefore, LOXL2 may promote tumor progression of glioma by synergistic multiple signaling pathways.

\section{Conclusion}

In conclusion, our data suggested that high LOXL2 expression indicated poor overall survival of glioma patients, and LOXL2 may exert tumor-promoting effects by enhancing Src and E-cadherin signaling pathways.

\section{Disclosure}

The authors report no conflicts of interest in this work.

\section{References}

1. Jansen M, Yip S, Louis DN. Molecular pathology in adult gliomas: diagnostic, prognostic, and predictive markers. Lancet Neurol. 2010; 9(7):717-726.

2. Shi H, Zhang S. Expression and prognostic role of orphan receptor GPR110 in glioma. Biochem Biophys Res Commun. 2017;491(2): 349-354.

3. Louis DN, Ohgaki H, Wiestler OD, et al. The 2007 WHO classification of tumours of the central nervous system. Acta Neuropathol. 2007; 114(2):97-109.

4. Johnson DR, Galanis E. Incorporation of prognostic and predictive factors into glioma clinical trials. Curr Oncol Rep. 2013;15(1):56-63.

5. Furnari FB, Fenton T, Bachoo RM, et al. Malignant astrocytic glioma: genetics, biology, and paths to treatment. Genes Dev. 2007;21(21): 2683-2710.

6. Verhaak RG, Hoadley KA, Purdom E, et al. Integrated genomic analysis identifies clinically relevant subtypes of glioblastoma characterized by abnormalities in PDGFRA, IDH1, EGFR, and NF1. Cancer Cell. 2010;17(1):98-110.

7. Cox TR, Erler JT. Remodeling and homeostasis of the extracellular matrix: implications for fibrotic diseases and cancer. Dis Model Mech. 2011;4(2):165-178.

8. Cano A, Santamaría PG, Moreno-Bueno G. LOXL2 in epithelial cell plasticity and tumor progression. Future Oncol. 2012;8(9):1095-1108.

9. Lopez KM, Greenaway FT. Identification of the copper-binding ligands of lysyl oxidase. J Neural Transm. 2011;118(7):1101-1109.

10. Cuevas EP, Moreno-Bueno G, Canesin G, Santos V, Portillo F, Cano A. LOXL2 catalytically inactive mutants mediate epithelial-to-mesenchymal transition. Biol Open. 2014;3(2):129-137.

11. Kim YM, Kim EC, Kim Y. The human lysyl oxidase-like 2 protein functions as an amine oxidase toward collagen and elastin. Mol Biol Rep. 2011;38(1):145-149.

12. Schietke R, Warnecke C, Wacker I, et al. The lysyl oxidases LOX and LOXL2 are necessary and sufficient to repress E-cadherin in hypoxiainsights into cellular transformation processes mediated by HIF-1. J Biol Chem. 2010;285(9):6658-6669.

13. Zheng W, Geng AQ, Li PF, Wang Y, Yuan XB. Robo4 regulates the radial migration of newborn neurons in developing neocortex. Cerebral Cortex. 2012;22(11):2587-2601.

OncoTargets and Therapy

\section{Publish your work in this journal}

OncoTargets and Therapy is an international, peer-reviewed, open access journal focusing on the pathological basis of all cancers, potential targets for therapy and treatment protocols employed to improve the management of cancer patients. The journal also focuses on the impact of management programs and new therapeutic agents and protocols on

Submit your manuscript here: http://www.dovepress.com/oncotargets-and-therapy-journa
14. Peng L, Ran YL, Hu H, et al. Secreted LOXL2 is a novel therapeutic target that promotes gastric cancer metastasis via the Src/FAK pathway. Carcinogenesis. 2009;30(10):1660-1669.

15. Park JS, Lee JH, Lee YS, Kim JK, Dong SM, Yoon DS. Emerging role of LOXL2 in the promotion of pancreas cancer metastasis. Oncotarget. 2016;7(27):42539.

16. Peinado H, Portillo F, Cano A. Switching on-off snail: LOXL2 versus GSK3? Cell Cycle. 2005;4(12):1749-1752.

17. Peinado H, Ballestar E, Esteller M, Cano A. Snail mediates E-cadherin repression by the recruitment of the $\operatorname{Sin} 3 \mathrm{~A} /$ histone deacetylase 1 (HDAC1)/HDAC2 complex. Mol Cell Biol. 2004;24(1):306-319.

18. Jourdan-Le Saux C, Tronecker H, Bogic L, Bryant-Greenwood GD, Boyd CD, Csiszar K. The LOXL2 gene encodes a new lysyl oxidase-like protein and is expressed at high levels in reproductive tissues. $J$ Biol Chem. 1999;274(18):12939-12944.

19. Moon HJ, Finney J, Ronnebaum T, Mure M. Human lysyl oxidaselike 2. Bioorg Chem. 2014;57:231-241.

20. Barker HE, Bird D, Lang G, Erler JT. Tumor-secreted LOXL2 activates fibroblasts through FAK signaling. Mol Cancer Res. 2013;11(11): 1425-1436.

21. Moreno-Bueno G, Salvador F, Martín A, et al. Lysyl oxidase-like 2 (LOXL2), a new regulator of cell polarity required for metastatic dissemination of basal-like breast carcinomas. EMBO Mol Med. 2011; 3(9):528-544

22. Peinado H, Olmeda D, Csiszar K, et al. A molecular role for lysyl oxidase-like 2 enzyme in Snail regulation and tumor progression. EMBO J. 2005;24(19):3446-3458.

23. Kasashima $H$, Yashiro M, Kinoshita $H$, et al. Lysyl oxidase-like 2 (LOXL2) from stromal fibroblasts stimulates the progression of gastric cancer. Cancer Lett. 2014;354(2):438-446.

24. Barker HE, Chang J, Cox TR, et al. LOXL2-mediated matrix remodeling in metastasis and mammary gland involution. Cancer Res. 2011; 71(5):1561-1572

25. Lin ZY, Chuang YH, Chuang WL. Cancer-associated fibroblasts up-regulate CCL2, CCL26, IL6 and LOXL2 genes related to promotion of cancer progression in hepatocellular carcinoma cells. Biomed Pharmacother. 2012;66(7):525-529.

26. Mizuno K, Seki N, Mataki H, et al. Tumor-suppressive microRNA-29 family inhibits cancer cell migration and invasion directly targeting LOXL2 in lung squamous cell carcinoma. Int J Oncol. 2016; 48(2):450-460.

27. Payne SL, Hendrix MJ, Kirschmann DA. Paradoxical roles for lysyl oxidases in cancer - a prospect. J Cell Biochem. 2007;101(6): 1338-1354.

28. Ropero S, Esteller M. The role of histone deacetylases (HDACs) in human cancer. Mol Oncol. 2007;1(1):19-25.

29. von Burstin J, Eser S, Paul MC, et al. E-cadherin regulates metastasis of pancreatic cancer in vivo and is suppressed by a SNAIL/ HDAC1/HDAC2 repressor complex. Gastroenterology. 2009;137(1): 361-371. e1-e5.

30. Millanes-Romero A, Herranz N, Perrera V, et al. Regulation of heterochromatin transcription by Snail1/LOXL2 during epithelial-tomesenchymal transition. Mol Cell. 2013;52(5):746-757.

31. Mitra SK, Schlaepfer DD. Integrin-regulated FAK-Src signaling in normal and cancer cells. Curr Opin Cell Biol. 2006;18(5):516-523.

patient perspectives such as quality of life, adherence and satisfaction The manuscript management system is completely online and includes a very quick and fair peer-review system, which is all easy to use. Visit http://www.dovepress.com/testimonials.php to read real quotes from published authors. 